Aus der Medizinischen Klinik der Akademie für praktische Medizin in Düsseldorf. (Direktor: Geheimrat Aug. Hoffmann.)

\title{
Zur Behandlung von Herzkranken mit Traubenzuckerinfusionen.
}

\section{Von Dr. W. Pfalz.}

Für die Pathologie des Herzens ist die von Büdingen aufgeworfene Fragestellung, ob das im Herzmuskel aufgespeicherte und das im Blute zugeführte Nährmaterial immer ausreichend ist, um dem Herzen die von ihm verlangte Arbeitsleistung zu ermöglichen, etwas Neues. Im allgemeinen wurde angenommen, daß im Blute stets genügend Nährstoffe vorhanden seien, um alle Organe ausreichend zu versorgen, und die Folgen gestörter Zirkulation wurden in erster Linie der unzureichenden $\mathrm{O}_{2}$-Zufuhr zugeschrieben. Büdinge ${ }^{1}$ ) zieht nun in Erwägung, ob nicht die Krankheitserscheinungen bei der Koronarsklerose z. B. zum großen Teil durch die ungünstige Herzernährung infolge der Gefäßverengerung bedingt sind und ob nicht auch anderweitig bedingte Störungen in der Nahrungszufuhr zum Herzen die Funktion des Herzmuskels ungünstig beeinflussen.

1) Vgl. seinen Aufsatz in dieser Wochenschrift. 
Er nimmt an, daß für die Tätigkeit dos Herzmuskels die drei Hauptgruppen der organischen Nährstoffe, Eiweiß, Fette und Kohlenhydrate, nicht gleichwertig sind, sondern da $\beta$, ebenso wie beim Skelettmuskel, in erster Linie die Kohlenhydrate, und zwar in Form von Traubenzucker (Glykose), als Energiequelle für. die Herzmuskelkraft benötigt werden. Der physiologische Gehalt des Blutes an Traubenzucker beträgt $0,07-0,11 \%$ (Ivar Bang), der wohl für den Bedarf des normal arbeitenden Herzens genügt. Da aber, ebenso wie bei der Skelettmuskulatur, beim Herzen oft rasche und unvermittelte Uebergänge von der geringsten bis zur höchsten Leistung vorkommen, so müssen Reservestoffe vorhanden sein, die je nach Bedarf als Energiequelle mitherangezogen werden können. Der wichtigste Reservestoff im Kohlenhydratstoffwechsel ist das Glykogen, dessen Vorkommen im spezifischen Muskelsystem des Herzens schon langer bekannt war, von Berblinger aber auch in den übrigen Myokardfasern, ahnlich wie in den Skelettmuskeln, in wechselnden Mengenverhaltnissen nachgewiesen wurde. Nach Buldingen sind nun Falle denkbar, wo die dem Herzen zugeführte Menge von Nahrungsstoffen, speziell von Traubenzucker, unzureichend ist, wo es nicht zu einer für den Bedarf genügenden synthetischen Bildung und Ablagerung von Glykogen im Herzmuskel kommt, kurz, wo eine Ernahrungsstörung des Herzmuskels, eine Kardiodystrophie vorliegt. Budingen unterscheidet im wesentlichen zwei Arten von Kardiodystrophien :

Die kardiogenen oder endogenen Kardiodystrophien deren nâchste Ursache und Wirkung im Herzen selbst liegt. Hierher gehört die Koronarsklerose, bei der die durch die Kranzarterien strömende Blutmenge, und damit auch die Menge des Blutzuckers, durch funktionell oder organisch bedingte Gefaßverengerung zeitweise oder dauernd geringer als normal ist. Weiter sind hierher die Falle zu rechnen, wo eine standige Mehrarbeit des ganzen Herzmuskels oder seiner Teile vorliegt, wo der normale Gehalt des Blutes an Traubenzucker infolge des erhöhten Bedarfs relativ ungenügend für die Herzernahrung wird (relative Hypoglykämie bei Blutdrucksteigerung mit Hypertrophie und Dilatation des Herzens, Myodegeneratio mit Herzerweiterung, Klappenfehlern mit zunehmender Stenosierung eines Ostiums).

2. Die ektokardiogenen oder ektogenen Kardiodystrophien, deren Ursache außerhalb des Herzens, deren Wirkung aber in ihm liegt. Als besonders wichtig erscheint Büdingen die absolute Verminderung des Blutzuckers (absolute Hypoglykamie), welche ein charakteristisches, bisher noch nicht erschlossenes Krankheitsbild, die „hypoglykamische Kardiodystrophie" bedingen soll, als deren Kardialsymptome außer der Hypoglykamie die Druckbeschwerden in der Herzgegend, die Herabsetzung der körperlichen Leistungsfahigkeit und die leisen Herztöne genannt werden. Hypothetisch sind zu erwăhnen Störungen der Zuckerverarbeitung im Herzmuskel bei Erkrankungen der Drüsen mit innerer Sekretion, bei Infektionskrankheiten, Vergiftungen und Altersvorgangen.

Die Kardiodystrophie in den oben kurz geschilderten Formen bildet nun nach Büdingen eine Indikation zur Behandlung mit hochprozentigen $(10-20 \%)$ Traubenzuckerinfusionen, die durch die plötzliche Ueberschwemmung des venösen Blutes mit Traubenzucker einen Năhrreiz auf das Herz ausüben und den Blutzuckerspiegel erhöhen sollen.

Wir haben die Behandlungsmethode an einer Reihe geeignet erscheinender Fälle nachgeprüft, und die Ergebnisse seien hier kurz mitgeteilt.

Die Technik der Infusionen ist relativ einfach. Wir benutzen eine 12-20\% ige Traubenzuckerlösung, die aus den chemisch reinen Merckschen Glykosetabletten hergestellt und vor dem Gebrauch sterilisiert wird. Es werden durchschnittlich sechs bi zehn Infusionen von je 200-300 ccm in einwöchentlichen $\mathrm{Ab}$ standen gemacht. Zur Infusion der körperwarmen Lösung bedienen wir uns des Infusionsapparates nach Dr. Schreiber, der aus einer Kristallglaskaratspritze, einer Dreiweghahnkanüle und einem graduierten Meßzylinder mit Schlauch besteht. Wegen Platzmangels muß ich auf die Wiedergabe der Krankengeschichten verzichten und möchte hier nur das Wesentlichste hervorheben. Von den mit Infusionen behandelten Herzkranken reagierten am besten zwei Falle von Koronarsklerose mit Angina pectoris ohne wesentliche Blutdrucksteigerung, bei denen am Schluß der $\mathrm{Be}$ handlung die anfangs bestehenden Beschwerden (Beklemmungen und Schmerzen in der Brust und im linken Arm nach Anstrengungen) fast völlig verschwunden waren. Auch in drei Fallen von allgemeiner Arteriosklerose mit Hypertonie und Angina pectoris und in einem Fall von peripherischer Arteriosklerose und Aortitis luetica mit starken Anfallen von Angina pectoris wirkten die Infusionen günstig insofern, als die Herzbeschwerden (Beklemmungen, Schmerzen in der Brust, Atemnot, Anfalle von Angina pectoris) wesentlich nachließen und die körperliche Leistungsfähigkeit sich hob. Bei einem Fall von Myodegeneratio cordis mit absoluter Unregelmaßigkeit infolge Vorhofflimmerns und Herzinsuffizienz wurde eine bedeutende Besserung der subjektiven Beschwerden und der Herzinsuffizienzerscheinungen erzielt, sodaß mäßige Anstrengungen wieder gut vertragen wurden. Die absolute Unregelmaßigkeit blieb in diesem Fall ebenso wie in zwei anderen Fallen bestehen, jedoch wurde die Aktion langsamer, und das anfangs bestehende Pulsdefizit kam zum Verschwinden. Auf einen weiteren Fall von Myodegeneratio cordis mit Herz- insuffizienz möchte ich etwas näher eingehen. Es handelte sich um ein dilatiertes Herz mit leisen Tönen, leisem systolischen Geräusch an der Spitze, beschleunigter, regelmäßiger Aktion; der Blutdruck war niedrig, der Puls klein. Es bestanden Oedeme und Stauungserscheinungen an den inneren Organen. Nach Digitalisdarreichung besserten sich die Beschwerden für kurze Zeit, dann aber traten wieder Dekompensationserscheinungen auf, schließlich Pulsus alternans. Es wurde der Versuch mit Traubenzuckerinfusionen gemacht, und ein gunstiger Einfluß auf den Krankheitsverlauf war nicht zu verkennen. Der Puls wurde wieder gleichmäßig, die Herztöne lauter und rein, die Anfälle von Atemnot wurden seltener. Besonders auffallend aber war die nach jeder Infusion auftretende Besserung, der Diurese. Wie aus der Urinkurve (s. Fig.), von.der wegen ihrer Lange nur ein charakte-

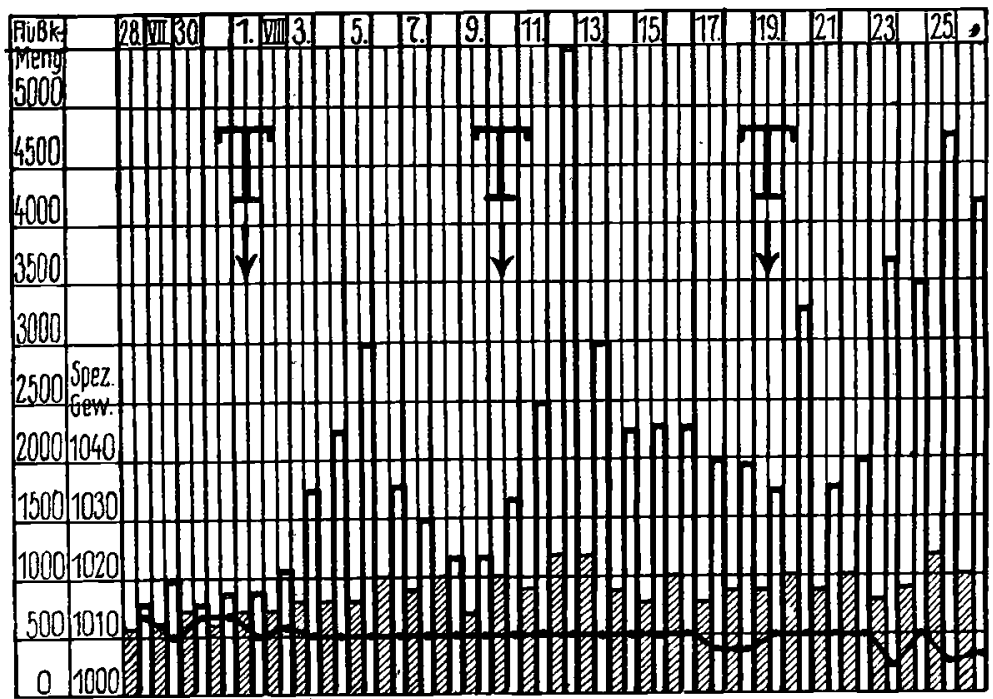

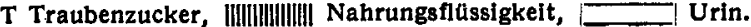

ristischer Teil wiedergegeben ist, ersichtlich, nahm die Urinausscheidung nach jeder Infusion für einige Tage deutlich $\mathrm{zu}$ und verringerte sich dann wieder, bis eine neue Infusion einen neuen Anstieg hervorrief. Nachdem im ganzen elf Infusionen gemacht waren, blieb die Urinausscheidung dauernd gut. Die Oedeme waren ganzlich verschwunden, und das Allgemeinbefinden hatte sich soweit gebessert, daß Patient schon wieder umhergehen konnte. Zu bemerken ist, daß während dieser Zeit keine Aenderung in der Medikation (kleine Dosen Digitalis, Theazylon) vorgenommen worden war. Vier Wochen spăter traten wieder Oedeme und Herzbeschwerden auf, und nun zeigte es sich, daß das Herz wieder auf Digitaliskörper gut reagierte. Dieser Fall ist deswegen besonders hervorzuheben, weil wir hier in dem Verhalten der Urinsekretion ein objektives Merkmal für die Besserung der Herzkraft durch die Infusionen haben. Eine Nierenwirkung, an die man auch denken könnte, glauben wir ausschließen zu können deshalb, weil die Diurese auch nach Aussetzen der Infusionen vier Wochen lang gut blieb. Auch das Verschwinden von Pulsus alternans und die Wiederherstellung der Anspruchsfăhigkeit auf Digitaliskörper sind objektive Anzeichen des gunstigen Einflusses der Traubenzuckerinfusionen.

Außer bei den oben erwâhnten Herzkrankheiten haben wir Infusionen auch bei Herzschwăche infolge langdauernder fieberhafter Erkrankungen angewandt; so bei einem Fall von ausgedehnter Pneumonie mit verzögerter Lösung. Bereits am vierten Krankheitstage waren rechter Unter- und Mittellappen sowie linker Unterlappen befallen, und der Allgemeinzustand des Kranken war sehr schwer. Von diesem Tage an wurden bis zu der erst am 26. Krankheitstage eintretenden Entfieberung taglich Infusionen (200 ccm einer $15 \%$ igen Traubenzuckerlösung) vorgenommen, die von dem Kranken subjektiv sehr angenehm empfunden wurden und auf die er seine allmăhliche Besserung bezog. Pulsfrequenz und Blutdruck blieben wăhrend dieser Zeit durchschnittlich auf derselben Höhe.

Bei einem anderen Fall von Pneumonie der ganzen rechten Lunge mit am siebenten Krankheitstage eintretender Herzschwache vermochte ein alsbald vorgenommener Aderlaß mit nachfolgender Traubenzuckerinfusion neben Adrenalin und Kampfer den Tod nicht aufzuhalten.

Soweit wir aus unserem Material Schlüsse ziehen können, läßt sich über die Wirkung der Traubenzuckerinfusionen zusammenfassend Folgendes sagen: Sie werden von den Kranken mit wenigen Ausnahmen subjektiv angenehm empfunden; die Herzschmerzen verschwinden häufig, Anfälle von Angina pectoris werden seltener. Damit einher geht häufig eine Hebung des Allgemeinzustandes, der körperlichen Leistungsfähigkeit. Am Herzen war in einem Fall ein Lauterwerden der Herztöne und ein Verschwinden von akzidentellen Geräuschen zu kon- 
statieren. Die Pulsfrequenz änderte sich nicht, ebensowenig der Blutdruck, dagegen wurde in einem Fall der Pulsus alternans zum Verschwinden gebracht und die Anspruchsfähigkeit des Herzens auf Digitalis wiederhergestellt. Wohl ebenfalls als objektive Wirkung der Infusionen ist die in dem oben erwähnten, mit Oedemen einhergehenden Fall konstatierte Besserung der Diurese zu bezeichnen. In den anderen Fällen fehlte ein sichtbarer Einfluß auf die Diurese. Zucker im Harn haben wir nach Infusionen nie beobachtet, was für eine gute Verwertung des infundierten Traubenzuckers im Organismus spricht.

Neben diesen oben geschilderten Wirkungen der Traubenzuckerinfusionen beobachteten wir eine Reihe von Nebenwirkungen. Relativ häufig, in sechs Fällen, traten nach Infusionen Fiebererscheinungen auf, und zwar stieg die Körpertemperatur meist mehrere Stunden nach der Infusion an auf $37,5-38,5^{\circ} \mathrm{C}$, nur in zwei Fällen über $39^{\circ} \mathrm{C}$, um dann entweder noch am selben Tage oder nach Ablauf von 24 Stunden zur Norm zurückzukehren. Da einige Patienten häufiger, andere selten oder garnicht Fiebererscheinungen bekamen und da diese Temperatursteigerungen auch von anderen Autoren beobachtet wurden, die ebenso wie wir selbstverständlich unter aseptischen Kautelen gearbeitet haben, so scheint es sich hier um eine nicht beherrschbare Nebenwirkung der Traubenzuckerinfusionen auf das Wärmezentrum nach Analogie des Salzfiebers zu handeln.

$B$ üdinge $n$ unterscheidet ein wahrscheinlich durch Fehler in der Asepsis bedingtes Frühfieber (innerhalb der ersten zwei Stunden) und ein Spătfieber (nach vier Stunden). Ob dieses letztere auf erhöhte Wärmebildung infolge Verbrennens des zugeführten Zuckers, wie Bernste in und Falta annehmen, zurückzuführen ist, oder ob es sich hier um vasomotorische Einflusse im Sinne $B$ üdingens handelt, müssen wir einstweilen dahingestellt sein lassen, möglicherweise wirkt beides zusammen.

Fieberlosen Frost beobachteten wir in zwei Fällen, Schüttelfrost und heftige Schmerzen in der Brust unmittelbar nach der Injektion in einem Falle; hier ist die Annahme einer vasomotorischen Reizung durch die hypertonische Lösung naheliegend, ebenso bei dem Auftreten von Schwindel während der Infusion. Die von Büdingen völlig rätselhaft genannten Schmerzen in der Kreuzbeingegend während und unmittelbar nach der Infusion, die bei drei seiner Patienten auftraten, konnten wir ebenfalls bei einem Patienten beobachten; sie wurden hier als direkt krampfartig bezeichnet.

Irgendwelche nachhaltigen Schädigungen sahen wir aber ebenso wie Büdingen in keinem Falle; im Gegenteil wurde von den Patienten immer wieder der wohltuende Einfluß der Infusionen gelobt.

Nach alledem kommen wir zu dem Resultat, daß die von Büdingen empfohlenen Traubenzuckerinfusionen bei manchen Herzkrankheiten unsere bisherige Therapie wertvoll unterstützen können.

Wie die günstige Wirkung der Traubenzuckerinfusionen zustandekommt, darüber können wir einstweilen noch kein abschließendes Urteil abgeben. Die Theorie Büdingens von den Kardiodystrophien und dem Einfluß des Traubenzuckers auf diese ist $\mathrm{zwar}$ sehr verlockend, aber nicht in allen Punkten stichhaltig. Eine länger dauernde Erhöhung des Blutzuckerspiegels wird durch die Infusion ja nicht erreicht (Ivar Bang), ebensowenig eine Aenderung der physikalischen Eigenschaften des Blutes (Lövy). Ferner können wir nach den Untersuchungen von Bernstein und Falta aus dem Traubenzuckergehalt des peripherischen venösen Blutes, welches ja für gewöhnlich zur Blutzuckerbestimmung benutzt wird, oder aus Glykogenbestimmungen der Organe keine sicheren Schlüsse auf den Kohlenhydratstoffwechsel im kardiopulmonalen Kreislauf machen, sondern wir müssen hierzu auch das Verhalten des respiratorischen Quotienten heranziehen. Und da zeigt es sich nun nach denselben Autoren, daß bei Ueberflutung des das rechte Herz durchströmenden Blutes mit Zucker der Ueberschuß in viel höherem Maße verbrannt als in Form von Glykogen aufgespeichert wird. Auch sind die anatomischen Befunde Berblingers über Glykogen im kranken und gesunden Herzmuskel durchaus nicht eindeutig, was von $B$ üd in gen zugegeben wird. Jedenfalls liegen die Verhältnisse hier sehr kompliziert, und es bedarf noch weiterer eingehender Untersuchungen, bevor wir uns über die Wirkungsweise der Traubenzuckerinfusionen ein klares Bild machen können.

Zusammenfassung. 1. Bei einer Reihe von Herzkrankheiten, bei denen $B$ üdingen eine "Kardiodystrophie" annimmt, wirken hochprozentige Traubenzuckerinfusionen günstig auf die subjektiven Beschwerden. In manchen Fällen finden sich auch objektive Symptome einer Besserung der Herzkraft.

2. Irgendwelche nachhaltigen Schädigungen durch Traubenzuckerinfusionen wurden bisher nicht beobachtet.

B e rblinger, Ziegler, Beitr. z. path. Anat. u. allg. Path. 55. 1912 S. 155. - Th. B ti dinge n, D. Arch. f. klin. Med. 114. 1914 S. 534. Ernährungsstörungen Traubenzuckerinfusionen Leipzigen zum Blutzucker und ihre Behandlung $m$ D. m. W. 1919 Nr. 3 S. 64. - B ernste in u. Fa it a D. Arch f klin. M. 125 . D. m. W. 1919 Nr. 8 S. $64 .-$ B ernste in u. Falta, D.
1918 S. $233 .-$ Ka usch, D. m. W. 1911 S. 8,1917 Nr. 23. 